\title{
Depth Analysis of Gradient in Teaching
}

\author{
Wenbing Wu, Rihua Liu \\ Nanchang Normal University, Nanchang, China
}

Correspondence to: Wenbing Wu, wwbysq@fjnu.edu.cn

Keywords: Gradient, Spatial Thinking, Quantitative Method

Received: November 4, $2021 \quad$ Accepted: December 7, 2021

Published: December 10, 2021

Copyright $\odot 2021$ by author(s) and Scientific Research Publishing Inc.

This work is licensed under the Creative Commons Attribution International License (CC BY 4.0).

http://creativecommons.org/licenses/by/4.0/

\section{Open Access}

\section{ABSTRACT}

Gradient and divergence are the basis of electromagnetic field theory, and have been a special difficulty in mathematical theory. Understanding these concepts requires strong spatial and abstract thinking. In this paper, through the graphical and quantitative methods, the significance of gradient representation is clearly displayed in the form of graphics.

\section{INTRODUCTION}

In the theory of electromagnetic field, in order to simplify the operation, some operator symbols are introduced. They have become indispensable tools in the field theory analysis. The Hamiltonian operator and Laplacian operator are widely used. Hamiltonian operator, the mathematical symbol is $\nabla$. In quantum mechanics, the Hamiltonian is observable, which corresponds to the total energy of the system. Corresponding to Hamiltonian operator, gradient and divergence in mathematics are abstract concepts: gradient is a vector, which indicates the direction that the directional derivative of a function at one given point reaches the maximum value, that is, the function changes fastest along the direction of gradient at the given point, and the rate of change is the largest (the modulus of the gradient). Gradient and divergence, as important mathematical tools, have been widely used in various aspects of scientific research, such as gene science [1], stochastic models [2], rectangular grids [3], interaction of population by temperature treatment [4], variational Inequalities [5]. This paper proposes a concrete understanding method for the concept of gradient. This method not only cultivates learners' spatial imagination, but also makes the abstract concept of gradient no longer difficult to understand.

\section{HOW TO UNDERSTAND THE GRADIENT}

\subsection{Directional Derivative and Gradient}

To understand the gradient, what the directional derivative is must be first grasped. The definition of directional derivative is given:

$$
\frac{\partial f}{\partial l}=\lim _{\rho \rightarrow 0} \frac{f(x+\Delta x, y+\Delta y)-f(x, y)}{\rho}=\frac{\partial f}{\partial x} \cos \varphi+\frac{\partial f}{\partial y} \sin \varphi .
$$


The above directional derivative refers to the rate of change of the function $z=f(x, y)$ along a straight line $l$. This straight line is actually the projection of the corresponding curve on curved surface $z=f(x, y)$ on the xoy plane. The vertical plane that completes the projection is called projection plane. Then, the directional derivative is actually the rate of change of the spatial curve along its own projection line on the xoy plane. Just as $\mathrm{d} y / \mathrm{d} x$ represents the rate of change of a curve in a 2-D plane along the $x$-axis at a certain point (that is, the slope of the tangent), referring to the explanatory diagram, you can clearly understand the meaning of directional derivative. The angle $\varphi$ is between the straight line $l$ and the $x$ axis in the xoy plane in Figure 1. When does the directional derivative reach its maximum value?

So, the definition of gradient is given:

Definition: Let the function $z=f(x, y)$ have a first-order continuous partial derivative in the plane area $\mathrm{D}$, then for every point, $\frac{\partial f}{\partial x} \boldsymbol{i}+\frac{\partial f}{\partial y} \boldsymbol{j}$ vector can be defined, it is called the gradient of function $z=f(x$, y), written as

$$
\operatorname{grad} f(x, y)=\frac{\partial f}{\partial x} \boldsymbol{i}+\frac{\partial f}{\partial y} \boldsymbol{j}
$$

Let $\boldsymbol{e}=\cos \varphi \boldsymbol{i}+\sin \varphi \boldsymbol{j}$ be the unit vector in direction $\boldsymbol{\imath}$

$$
\begin{aligned}
\frac{\partial f}{\partial l} & =\frac{\partial f}{\partial x} \cos \varphi+\frac{\partial f}{\partial y} \sin \varphi=\left\{\frac{\partial f}{\partial x}, \frac{\partial f}{\partial y}\right\} \cdot\{\cos \varphi, \sin \varphi\} \\
& =\operatorname{grad} f(x, y) \cdot \boldsymbol{e}=|\operatorname{grad} f(x, y)| \cos \theta,
\end{aligned}
$$

where $\theta=(\operatorname{gradf}(x, y), \boldsymbol{e})$.

When $\cos (\operatorname{grad} f(x, y), \boldsymbol{e})=1, \frac{\partial f}{\partial l}$ reaches its maximum.

Obviously, the maximum value of directional derivative is gradient.

\subsection{Explanation of Gradient}

With the above knowledge, the meaning of gradient can be explained in detail here. Suppose there is a conical curved surface in Figure 2. This surface is the function $f(x, y)$. Now intercept the surface with a plane, and then get the curve in Figure 3. Then raise the plane, intercept multiple curves, and project them on the xoy plane to get Figure 4.

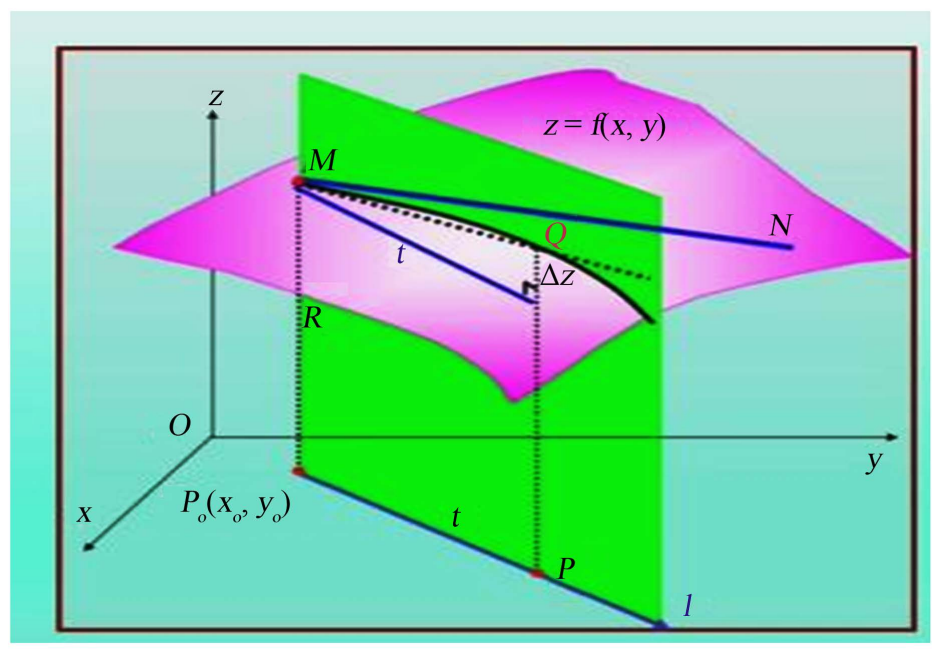

Figure 1. Illustration of directional derivative. 


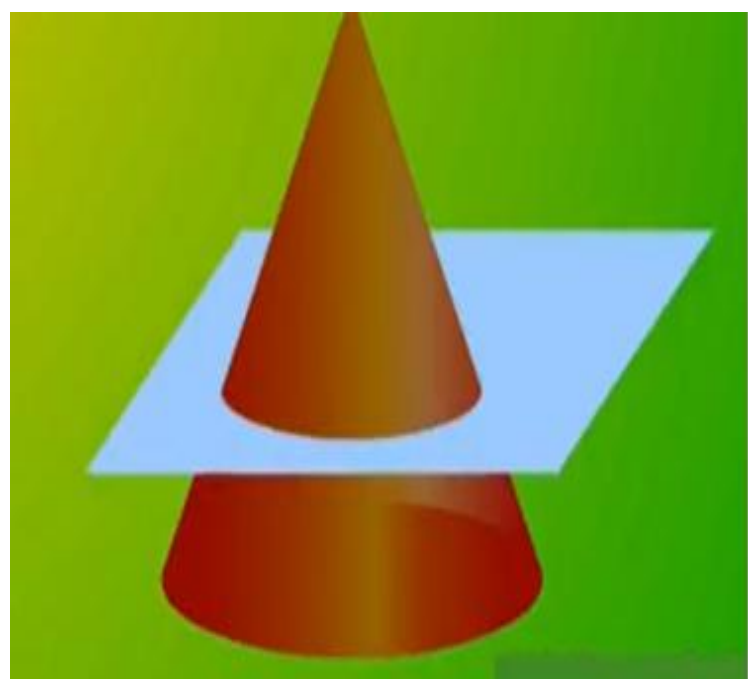

Figure 2. Cone and section.

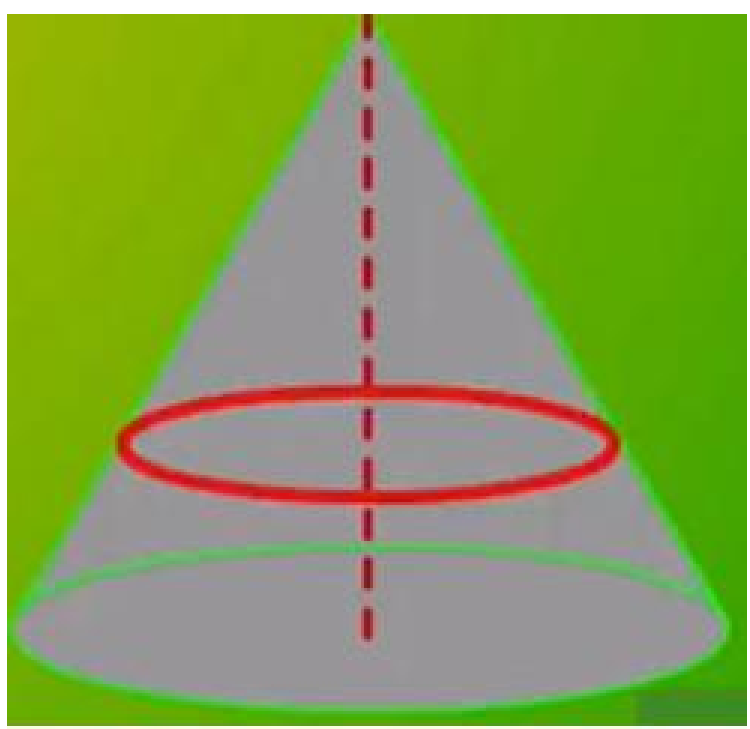

Figure 3. Cone is intercepted.

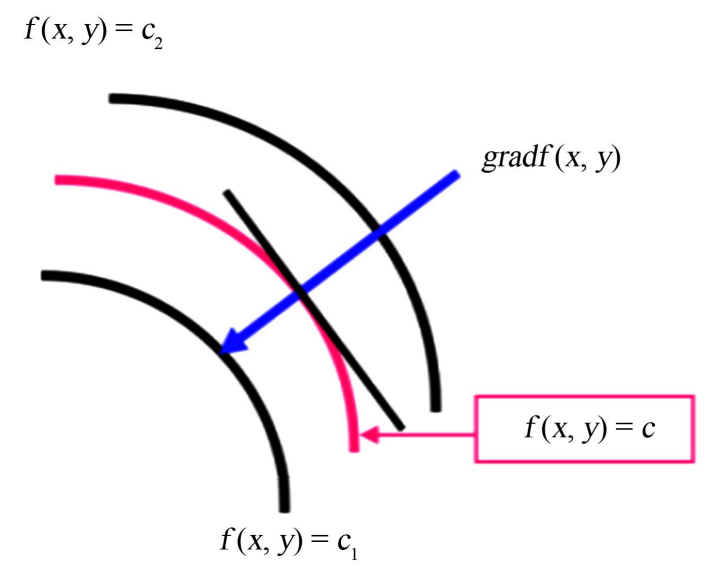

Figure 4. Contour map. 
It can be seen that all the curves $f(x, y)=c$ intercepted are contour lines, where the larger the $c$, the higher the height; the higher the intercepting plane, the smaller the intercepted circle. In Figure 4, $c_{1}>c_{2}$. If $c$ is continuously changing, then the entire surface is projected onto the xoy plane. So, how to explain the judgement "gradient is the direction with the fastest height change"? It can be seen from the definition of directional derivative that gradient is the value when directional derivative reaches its maximum value, that is, when the ratio reaches its maximum value, it is of course the direction with the fastest height change. Then why the direction of gradient is the normal direction of one certain point in the contour? Note that point $\mathrm{P}$ corresponds to one point on the conical surface, the problem is described as follows:

$$
\frac{\partial f}{\partial l}=\lim _{\rho \rightarrow 0} \frac{f(x+\Delta x, y+\Delta y)-f(x, y)}{\rho} .
$$

Let the expression of a surface be $z=f(x, y)$, then the contour line is $\left\{\begin{array}{l}z=f(x, y) \\ z=c\end{array}\right.$, that is, $f(x, y)=c$, the slope at any point on the contour line is: $\mathrm{d} y / \mathrm{d} x$, and $z=f(x, y)$ indicates that the slope on the contour line is $\frac{\mathrm{d} y}{\mathrm{~d} x}=\frac{\partial f}{\partial x} / \frac{\partial f}{\partial y}$, the normal direction corresponding to the point is the negative reciprocal of the slope, that is $-\frac{1}{\frac{\mathrm{d} y}{\mathrm{~d} x}}=-\frac{\mathrm{d} x}{\mathrm{~d} y}=-\frac{\partial f}{\partial y} / \frac{\partial f}{\partial x}=\tan \theta$, the angle $\theta$ is between the normal and the $x$-axis. If the direction of gradient is the same as the normal of contour, the vertical relationship is proved.

Finally, gradient can be understood by spatial imagination: Gradient is to help a person standing on the hillside (point $\mathrm{P}$ ) to find the shortest path to the top of mountain. So, how can relate contour maps to this problem? In order to solve this problem, it is necessary to establish a 3-D rectangular coordinate system. The xoy plane is the plane at the bottom of the slope, and the $Z$ axis is the straight line perpendicular to the $x o y$ plane where point $\mathrm{P}$ is located. That is to say, the origin of the coordinate system is the projection of point $\mathrm{P}$ on the $x$ oy plane. When the directional straight line $l$ (including the projection plane in Figure 1) rotates around the origin ( $Z$-axis in Figure 5), a spatial curve must be intercepted on the conic surface (hillside), as shown in Figure 5. This curve is the trajectory of the person standing on the hillside

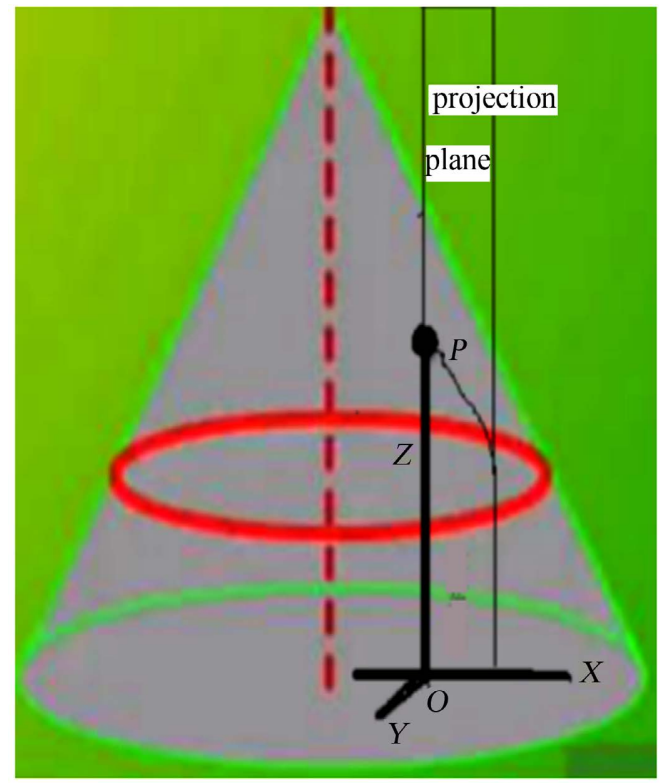

Figure 5. Projection plane of cone. 
moving in any direction. When the direction line turns to be consistent with the normal direction of point $\mathrm{P}$ in the contour map, the direction line finds the direction of gradient (note that the directional line and gradient vector are always in xoy plane at the same time). At this time, the space curve intercepted by the projection plane on the hillside must connect the $\mathrm{P}$ point where the person stands and the top of the slope. If not, it is not the gradient direction, and this curve is the shortest path for the person to reach the top of the mountain (not the foot of the mountain, which represents the opposite direction of gradient). It can be seen that the gradient is actually the projection of the shortest path to be found on the conic surface on the xoy plane. Then, every step the person takes to the top of the mountain along this track is equivalent to crossing from one contour line to the adjacent one along the gradient direction in the contour map. In short, gradient is a vector whose value is equal to the maximum value of directional derivative, and its direction is the normal direction of the given point in the contour map.

\section{CONCLUSION}

Gradient is a basic concept in mathematics, and it has a wide range of applications in fields such as field theory. It is also a difficult point in teaching. On the basis of directional derivative, a cone being intercepted is imagined, and through the scene of a person climbing to the top of a mountain, the concept of gradient is clearly explained. The method provided in this article provides an idea for gradient teaching.

\section{FUNDING}

This paper is supported by Research Foundation of the Nanchang Normal University for Doctors (NSBSJJ2018014). Research project of major education and teaching reform in Fujian Province (FBJG20190284).

\section{CONFLICTS OF INTEREST}

The authors declare no conflicts of interest regarding the publication of this paper.

\section{REFERENCES}

1. Allen, S.L., et al. (2017) Sex-Biased Transcriptome Divergence along a Latitudinal Gradient. Molecular Ecology, 26, 1256-1272. https://doi.org/10.1111/mec.14015

2. Khan, M.E. (2016) Faster Stochastic Variational Inference Using Proximal-Gradient Methods with General Divergence Functions. Journal of Comparative Neurology, 319, 359-386.

3. Hyman, J.M. and Shashkov, M. (1997) Natural Discretizations for the Divergence, Gradient, and Curl on Logically Rectangular Grids. Computers \& Mathematics with Applications, 33, 81-104. https://doi.org/10.1016/S0898-1221(97)00009-6

4. Eldon, J., Bellinger, M.R. and Price, D.K. (2019) Hawaiian Picture-Winged Drosophila Exhibit Adaptive Population Divergence along a Narrow Climatic Gradient on Hawaii Island. Ecology and Evolution, 9, 2436-2448. https://doi.org/10.1002/ece3.4844

5. Semenov, V.V. (2018) Modified Extragradient Method with Bregman Divergence for Variational Inequalities. Journal of Automation and Information Sciences, 50, 26-37. https://doi.org/10.1615/JAutomatInfScien.v50.i8.30 